\title{
CRIAÇÃO DO BANCO DE DADOS DA MICOTECA DO LABORATÓRIO DE QUÍMICA E BIOMOLÉCULAS (LAQUIBIO) DO ISECENSA
}

\author{
Vicente Mussi-Dias $^{1,2^{*}}$, Davi Couto Fernandes ${ }^{3}$ \& Maria das Graças Machado Freire ${ }^{1}$
}

MUSSI-DIAS, V., FERNANDES, D.C., FREIRE, M.G.M. Criação do banco de dados da micoteca do Laboratório de Química e Biomoléculas (LAQUIBIO) do ISECENSA. Perspectivas Online: Biológicas \& Saúde, v.8, n.28, p. 92-102, 2018.

\section{RESUMO}

Os fungos estão amplamente presentes em todos os ecossistemas da terra e, embora essa distribuição não seja ainda bem documentada, esforços têm sido feitos para catalogar e identificar estes organismos em diversos ambientes. Um ecossistema praticamente inexplorado quanto à micoflora é o de restinga, fortemente presente no norte do estado do Rio de Janeiro. A busca por fungos de plantas das restingas fluminenses tem sido feita desde 2012 pela equipe do Laboratório de Química e Biomoléculas (LAQUIBIO) do ISECENSA. Neste período, mais de 2000 isolados fúngicos foram obtidos e estão sendo mantidos conservados para pesquisas e utilização futura. Assim, este trabalho teve como objetivo a criação do banco de dados da
Micoteca do LAQUIBIO, incluindo imagens das colônias de cada isolado para fins de consulta e comparação. Os fungos foram purificados e armazenados, recebendo numeração e os dados de coleta mantidos em planilha eletrônica. Foram feitas fotografias das colônias crescidas em placas de Petri e associadas à planilha, compondo o banco de dados. A análise dos resultados permitiu o agrupamento dos fungos a fim de permitir uma busca rápida, a seleção por restinga de origem, planta hospedeira e identificação morfológica ou molecular, bem como um registro permanente das características das colônias para comparação com fins de identificação e divulgação dos espécimes.

Palavras-chave: Biodiversidade; Preservação, Micologia, Restinga. 


\begin{abstract}
Fungi are widely present in all earth's ecosystems and, although this distribution is not yet well documented, efforts have been made to catalog and identify these organisms in various environments. Restinga is a practically unexplored ecosystem with regard to its mycoflora, strongly present in the north of the Rio de Janeiro state. The search for fungi on plants in the Rio de Janeiro restingas has been done since 2012 by the group of the Laboratory of Chemistry and Biomolecules (LAQUIBIO) of ISECENSA. During this period, more than 2000 fungal isolates were obtained and are being kept for further research and use. Thus, this work had as

Micoteca database, including colonies images of each isolate for the purpose of consultation and comparison. The fungi were purified and stored, receiving numbering and the collection data kept in a worksheet. Photographs were taken of the colonies grown on Petri plates and associated with the spreadsheets, composing the database. The analysis of the results allowed the grouping of the fungi in order to allow a quick search, selection by restinga source, host plant and morphological or molecular identification, as well as a permanent record of the colonies characteristics for comparison aiming identification and specimens dissemination.
\end{abstract} objective the creation of the LAQUIBIO

Keywords: Biodiversity, Preservation, Mycology, Restinga

\footnotetext{
${ }^{1}$ Institutos Superiores de Ensino do CENSA - ISECENSA - Laboratório de Química e Biomoléculas - LAQUIBIO - Rua Salvador Correa, 139, Centro, Campos dos Goytacazes, RJ, CEP: 28035-310, Brasil;

${ }^{2}$ Universidade Estadual do Norte Fluminense Darcy Ribeiro - UENF - Laboratório de Entomologia e Fitopatologia - LEF/CCTA Av. Alberto Lamego, 2000, Parque Califórnia, Campos dos Goytacazes, RJ, CEP: 28013-602, Brasil;

${ }^{3}$ Aluno bolsista PIBIC/ISECENSA - Centro de Pesquisa e Pós-graduação - CPPG

(*) e-mail: vimdias@yahoo.com.br

Data de recebimento:07/12/2018 Aceito para publicação: 19/12/2018
} 


\section{INTRODUÇÃO}

A diversidade microbiana ainda é vista como invisível, embora para alguns setores como a ciência e a indústria apresente interesse e implicações econômicas (SANTOS e LIMA, 2001). A proteção do meio ambiente e a preservação da biodiversidade é uma das recomendações apontadas pelas Nações Unidas, no que se refere ao potencial genético, inclusive dos microrganismos, para aplicação na agricultura, saúde e bem-estar, o que acarreta a necessidade de preservação dos mesmos (GOLDMAN e TALLIS, 2009).

Localizados no extremo norte do estado do Rio de Janeiro, os municípios de São Francisco do Itabapoana, Campos dos Goytacazes, São João da Barra e Macaé apresentam fitofisionomia diversificada, sendo apontada como importante área de transição florestal da costa do Sudeste brasileiro na qual pode se encontrar diversificação de ecossistemas, dentre eles as Restingas (OLIVEIRA-FILHO e FONTES, 2000).

A partir de uma visão preservacionista voltada aos recursos naturais da região Norte Fluminense (FREIRE et al., 2015) pôde-se vislumbrar uma fonte inesgotável de fungos endofíticos disponíveis nas Restingas da região e, dessa forma, unindo a diversidade vegetal local aos interesses da prospecção micológica para fins científicos foi estabelecida a Micoteca de fungos endofíticos na nossa região (MUSSI-DIAS et al., 2016; FREIRE et al., 2016). Várias definições são atribuídas a fungos endofíticos (HYDE e SOYTONG, 2008), dentre elas áquela que considera os fungos que vivem no interior da planta sem causar danos aparentes, ou seja, não sendo epifíticos ou fitopatogênicos em nenhum momento do seu ciclo de vida (AZEVEDO et al., 2000), embora alguns autores considerem a possibilidade de parasitismo (DHANYA e PADMAVATHY, 2014), principalmente no final do ciclo da cultura ou senescência natural da planta.

Esses microrganismos podem ser potencialmente úteis na agricultura e na indústria, sobretudo na farmacêutica e de defensivos agrícolas. Em consequência de constituírem-se potenciais substitutos aos produtos químicos, e exercerem ações de biocontrole e/ou promoção de crescimento de plantas favorecendo a preservação do ambiente, vêm sendo apontados como alternativas viáveis para sistemas de produção agrícolas, ecológica e economicamente sustentáveis (SANTOS e VARAVALLO, 2011).

Com a preservação em micoteca, cada espécie poderá ser utilizada de acordo com a sua atividade biológica, seja em pesquisas para o controle de pragas e patógenos ou para o benefício do homem na produção de substâncias úteis (BEZERRA et al., 2017), bem como para aplicação na natureza em biorremediação de poluentes ambientais (ANDRADE et al., 2015).

Coleções de cultura de fungos funcionam como fonte valiosa na recorrência do material preservado, sem a necessidade de buscá-lo novamente na natureza, sendo um repositório seguro à longo prazo. Além disso, elas têm funções fundamentais relacionadas à biodiversidade e biossistemática dos organismos (VIEIRA et al., 2018), pois os isolados depositados são a base para a maior parte do conhecimento real da diversidade e representam material de arquivo para o futuro (ABREU e TUTUNJI, 2004) além de serem avaliados por diferentes técnicas de preservação que permitirão avanços nos estudos biológicos (PASSADOR et al., 2010).

Para se ter um controle efetivo de cada fungo armazenado em uma coleção, há necessidade de criação de um banco de dados completo, com informações sobre origem, substrato ou associação em que foi encontrado até a identificação morfológica ou molecular, visando catalogar e facilitar 
sua utilização a qualquer momento, em função do interesse ao qual ele poderá ser aplicado (SILVESTRE, 2013). Assim, o principal objetivo deste trabalho foi preparar um banco de dados para agrupar e catalogar todos os fungos mantidos na Micoteca do LAQUIBIO, em planilha eletrônica, contendo informações relevantes de cada isolado, bem como vincular por fotodocumentação cada colônia por meio de imagens para fins de busca, comparação, identificação e divulgação dos espécimes.

\section{METODOLOGIA}

O isolamento dos fungos em laboratório, a partir de seus substratos (plantas, solo, liteira), foi realizado em meios de culturas padrão ou específico de acordo com a origem. Após sucessivas repicagens para limpeza e purificação das colônias, as mesmas foram observadas em microscópio de luz e, quando possível, identificadas ao nível de gênero. Cada cultura, devidamente identificada foi também armazenada por diferentes métodos, a fim de se avaliar a sobrevivência de cada isolado. Os métodos de preservação utilizados na Micoteca do LAQUIBIO são: discos de cultura submersos em água destilada esterilizada (método Castellani), meio de cultura inclinado em tubo de ensaio sob refrigeração a $7^{\circ} \mathrm{C}$ (ALFENAS e MAFIA, 2007), colônias em placas e discos de colônia em frascos eppendorf, ambos congelados a ${ }^{-} 18^{\circ} \mathrm{C}$.

Para o preparo do banco de dados foram feitas planilhas eletrônicas no programa Excel onde cada isolado recebeu uma numeração de registro e dados complementares (nome da restinga de origem, coordenadas geográficas, nome da planta hospedeira e da família botânica, data da coleta e do isolamento do fungo, parte da planta de onde foi isolado, características da colônia e identificação morfológica e/ou molecular).

Com a planilha completa procedeu-se agrupamentos em função da ordenação alfabética seguindo-se qualquer coluna selecionada. A partir daí, tornou-se possível escolher a busca por nome do fungo, nome da planta hospedeira, família botânica ou restinga de origem.

Ao mesmo arquivo foram associadas as fotografias dos isolados. Para tanto, quando os fungos, após isolamento encontravam-se em condições axênicas, em placas de Petri, cada colônia foi fotografada individualmente utilizando-se Câmera Digital Canon EOS Rebel T5i de 18.0 Megapixels. As fotografias foram numeradas a partir da planilha contendo a identificação dada a cada isolado de fungo, mantidas e agrupadas após o tratamento de contraste, luminosidade, tamanho e nitidez, criando-se um banco de imagens das colônias para fins de comparação e identificação.

Procedeu-se então o agrupamento das imagens na forma de um Catálogo de Fungos criado a partir dos Softwares PowerPoint ${ }^{\circledR}$, Adobe ${ }^{\circledR}$ Photoshop ${ }^{\circledR}$, CorelDRAW $\AA$, e Adobe ${ }^{\circledR}$ InDesign ${ }^{\circledR}$ específicos para tratamento e manipulação de imagens.

\section{RESULTADOS E DISCUSSÃO}

As pesquisas envolvendo fungos de plantas e do ambiente que vem sendo desenvolvidas no Laboratório de Química e Biomoléculas (LAQUIBIO) tem gerado um alto volume de dados e de isolados fúngicos. Isso possibilitou a criação de uma Micoteca, na qual são armazenados e preservados mais de 2000 isolados destes organismos (FREIRE et al., 2016).

A construção do banco de dados proveniente da Micoteca do LAQUIBIO possibilitou organizar e protocolar cada isolado de fungo armazenado (Figura 1). 


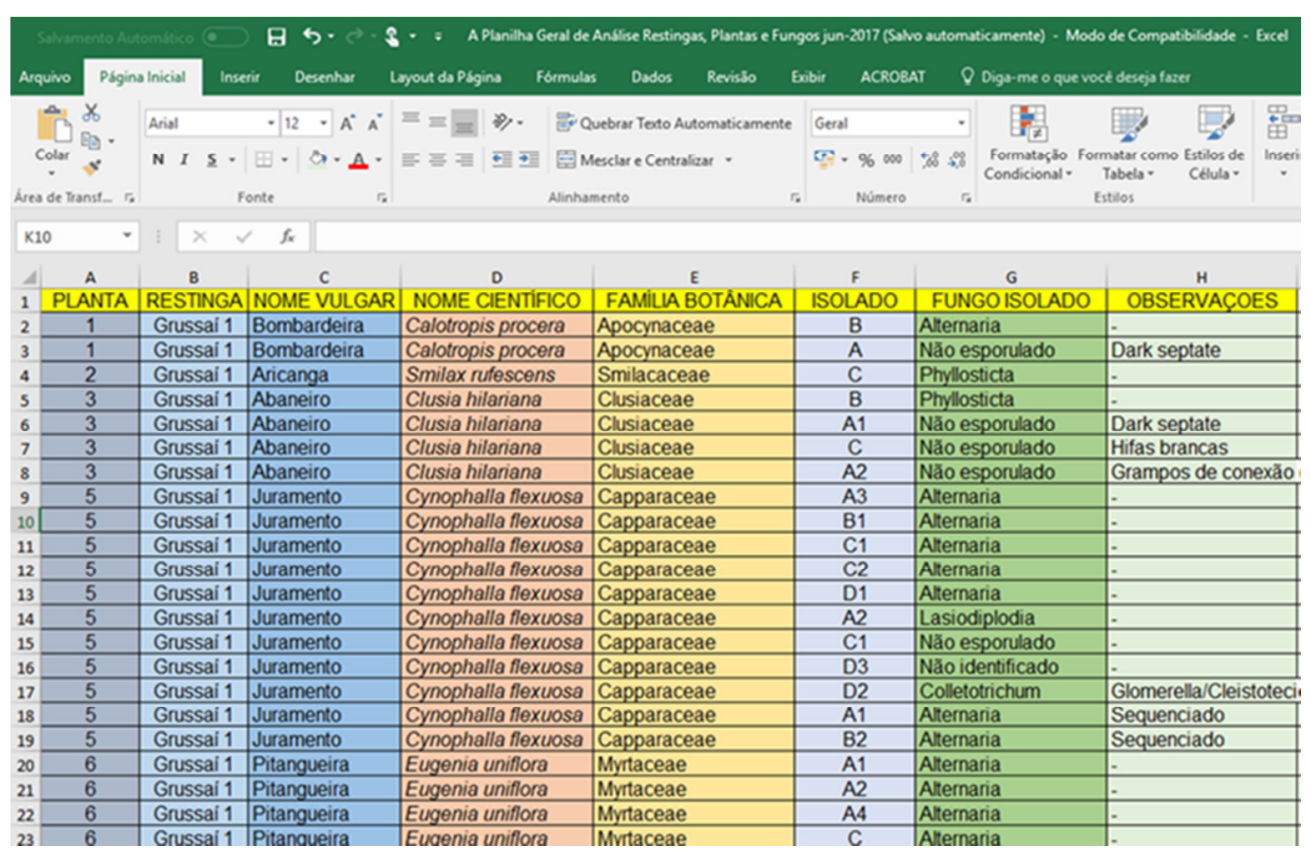

Figura 1: Modelo de planilha eletrônica utilizada para o gerenciamento da Micoteca do Laboratório de Química e Biomoléculas (LAQUIBIO) do ISECENSA, contendo dados de origem e identificação dos fungos endofíticos e das plantas coletas nas restingas fluminenses.

A organização sequencial dos fungos em planilha permitiu manter as características e identificação dos dados relacionados aos fungos de forma permanente para consulta. Isso facilita a busca por determinado isolado, assim como agrupamentos de plantas hospedeiras ou restinga de origem.

Também foi possível determinar o número de isolados e gêneros identificados em cada uma das quatro restingas estudadas (Figura 2). 


\begin{tabular}{|c|c|c|c|}
\hline Restinga de GRUSSAI & Restinga de GARGAÚ & Restinga do ACU & Restinga de JURUBATIBA \\
\hline Alternaria & Alternaria & Acremonium & Alternaria \\
\hline Aspergillus & Aspergillus & Alternaria & Aspergillus \\
\hline Chaetomium & Cladosporium & Asperglllus & Cladosporium \\
\hline Cladosporium & Cochliobolus & Cladosporium & Colletotrichum \\
\hline Colletotrichum & Colletotrichum & Colletotrichum & Curvularia \\
\hline Curvularia & Diaporthe & Curvularia & Exserohilum \\
\hline Fusarium & Eurotium & Exserohilum & Fusarium \\
\hline Helminthosporium & Glomerella & Fusarium & NE fungo \\
\hline Lasiodiplodia & Guignardia & Helminthosporium & NI fungo \\
\hline Lentinus & Helminthosporium & Macrophoma & Nigrospora \\
\hline NE fungo & NE fungo & Mortierella & Penicillium \\
\hline Neofusicoccum & NI fungo & NE fungo & Periconia \\
\hline NI fungo & Nigrospora & NI fungo & Pestalotiopsis \\
\hline Nigrospora & Penicillium & Nigrospora & Phoma \\
\hline Penicillium & Periconia & Penicillium & Phomopsis \\
\hline Pestalotiopsis & Pestalotiopsis & Periconia & Phyllosticta \\
\hline Phanerochaete & Phaeobotryosphaeria & Pestalotiopsis & \\
\hline Phoma & Phomopsis & Phoma & \\
\hline Phomopsis & Phyllosticta & Phomopsis & \\
\hline Phyllosticta & Stenella & Phyllosticta & \\
\hline Rhinocladiella & Talaromyces & Stemphylium & \\
\hline Setosphaeria & & Torula & \\
\hline Trichoderma & & & \\
\hline
\end{tabular}

Figura 2: Agrupamento de fungos da Micoteca do Laboratório de Química e Biomoléculas (LAQUIBIO) do ISECENSA, segundo a identificação por gênero, oriundos de plantas de quatro diferentes restingas do norte fluminense, RJ. NE e NI são fungos não esporulados e não identificados, respectivamente.

Registro semelhante foi realizado pelo Instituto Nacional de Pesquisas da Amazônia (INPA) preocupado em divulgar os macrofungos da Amazônia onde encontra-se uma das maiores diversidades de espécies de cogumelos do mundo (VARGAS-ISLA et al., 2014).

A partir destes agrupamentos também foi possível fazer correlações para o fornecimento de dados estatísticos a respeito da diversidade entre as espécies de fungos e as plantas hospedeiras (Figura 3), além de variações existentes dentre as áreas de restingas. O cruzando das informações viabiliza a escolha dos grupos de fungos a serem trabalhados em função do interesse de cada pesquisa, definido pela frequência de cada isolado ou da ocorrência dos mesmos em uma ou mais espécies vegetais.

Como exemplo prático, manipulando-se a busca entre as colunas da planilha do banco de dados foi possível detectar a presença de 56 isolados de fungos provenientes de pitangueira (Eugenia uniflora L.), 57 isolados de aroeira (Schinus terebinthifolia Raddi) e 42 isolados de batateira da praia (Ipomoea pes-caprae (L.) R. Br.). Os fungos do gênero Alternaria, Nigrospora, Pestalotiopsis, Phyllosticta e um isolado não esporulado aparecem sendo comuns para estas três espécies vegetais (Figura 4), indicando variabilidade no relacionamento planta-fungo, além de permitir buscas direcionadas, intra e inter-relacionadas. 


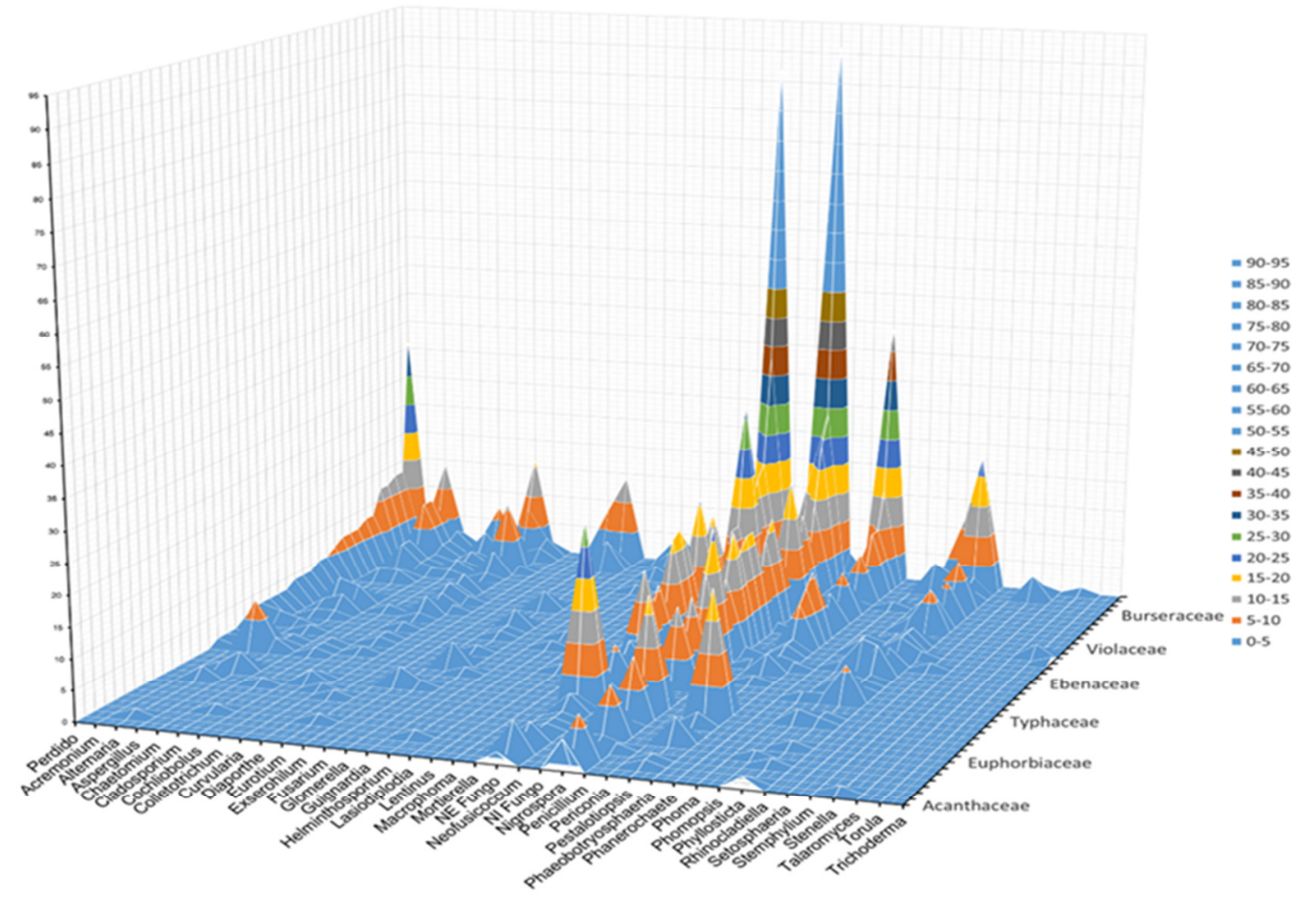

Figura 3: Quantitativo e diversidade de isolados fúngicos obtidos de restinga em função da família botânica das espécies vegetais estudadas. Dados obtidos a partir de planilha eletrônica da Micoteca do Laboratório de Química e Biomoléculas (LAQUIBIO) do ISECENSA.
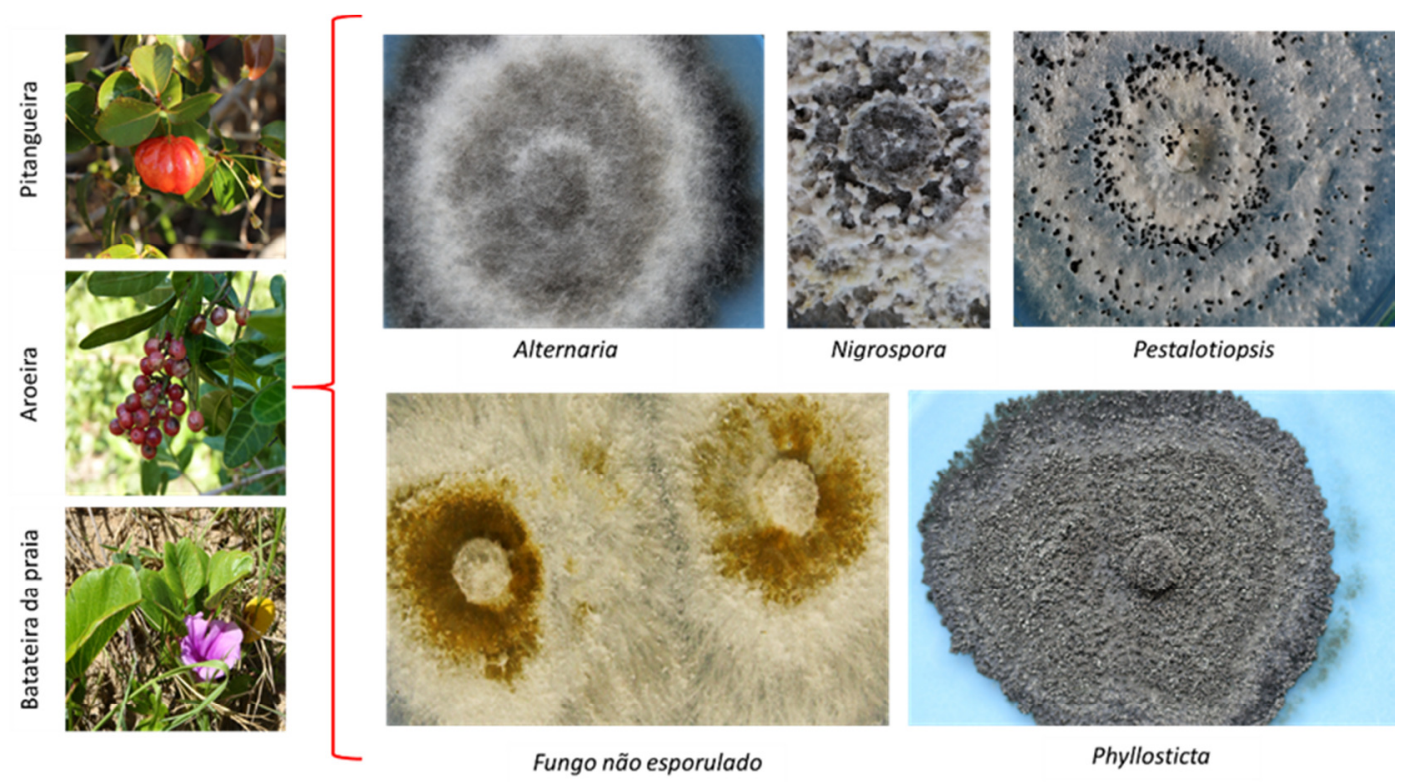

Figura 4: Variabilidade encontrada entre fungos endofíticos e espécies vegetais de quatro diferentes restingas do norte fluminense, RJ. 
A criação do banco de registro fotográfico das colônias dos fungos gerou um arquivo com mais de 6.000 imagens. Estas imagens foram tratadas e receberam a mesma numeração destinada ao banco de dados de cada colônia fúngica identificada anteriormente e registrada na planilha eletrônica da Figura 1. Dessa forma, foi possível manter o vínculo da imagem e das características de cada colônia com a identificação do respectivo isolado já protocolado na Micoteca (Figura 5). Isso criou uma identidade visual do isolado fazendo com que nas repicagens sucessivas ou recorrentes haja a possibilidade de comparar a fotografia registrada com a nova colônia ativada ou repicada, garantindo assim maior rapidez e confiabilidade no fornecimento e utilização dos isolados armazenados. Como parte do patrimônio natural ex-situ, as micotecas detém grande valor para efetivar projetos de pesquisa que incluem linhas de estudo com fármacos, controle ambiental e biotecnologia (CÂMARA et al., 2008).

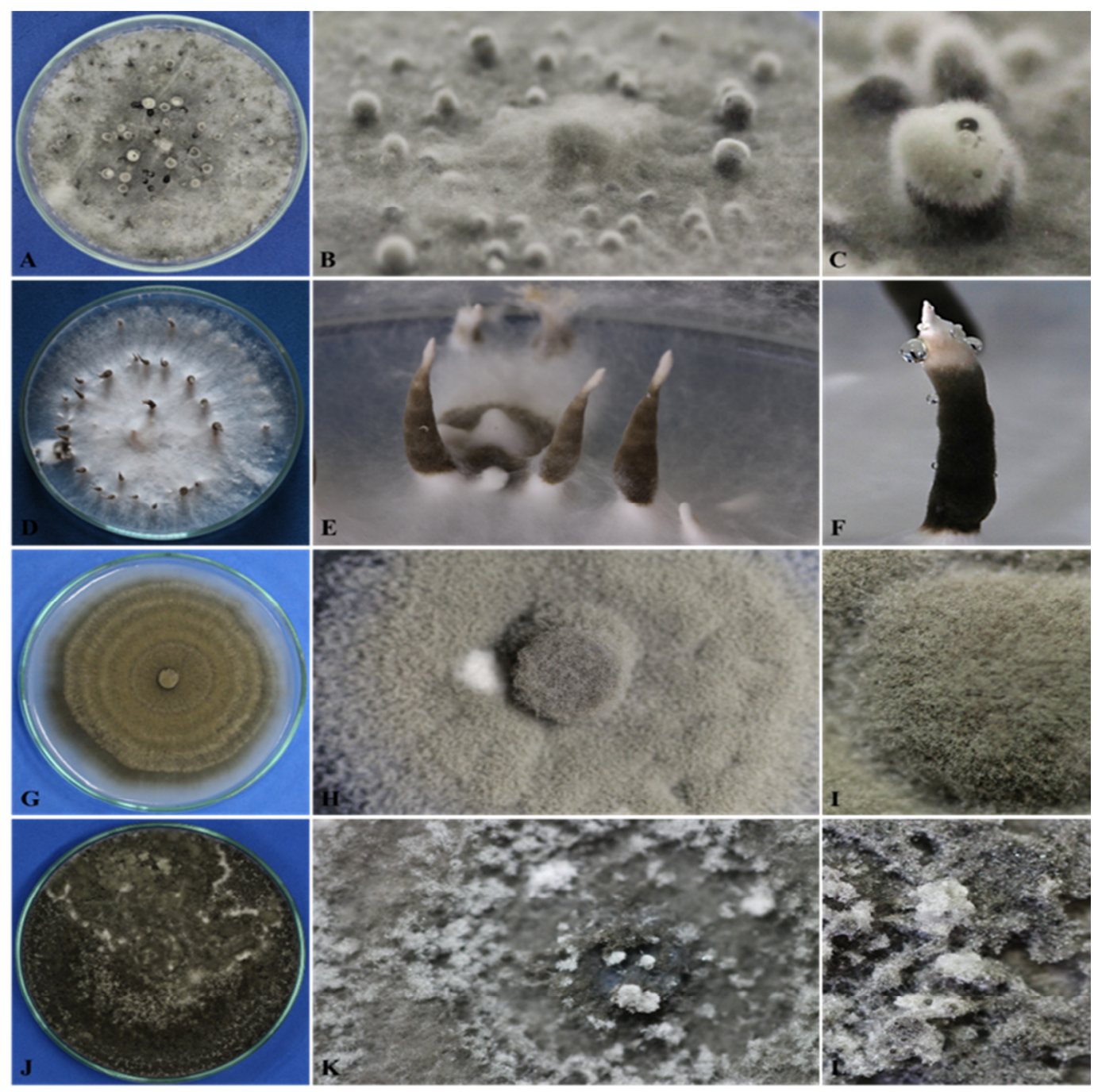

Figura 5: Registro fotográfico das colônias de fungos mantidos pela Micoteca do Laboratório de Química e Biomoléculas (LAQUIBIO). Coluna da esquerda são as colônias cultivadas em placas de Petri, ao centro a aparência mais aproximada do micélio e, à direita detalhes do isolado. A, B, C) Lasiodiplodia sp.; D, E, F) fungo não esporulado; G, H, I) Cladosporium sp.; J, K, L) Nigrospora $\mathrm{sp}$.

Persp. online: biol. \& saúde, Campos dos Goytacazes, 28 (8),92-102, 2018 
A partir do estabelecimento da planilha eletrônica e do banco de fotografias foi possível gerar o agrupamento dos dados aqui denominado de Catálogo dos Fungos Endofíticos da Micoteca do LAQUIBIO, no qual será possível apresentar a identidade desses fungos, na forma de imagens das colônias produzidas e cultivadas e, assim, dar início a um inventário e divulgação de imagens sobre essa diversidade desconhecida e ainda pouco explorada na nossa região.

Para ressaltar a importância da divulgação deste banco de dados, cita-se o trabalho de Gonçalves et al. (2017) que teve como objetivo a prospecção de patentes relacionadas aos fungos endofíticos aplicados à indústria farmacêutica. A pesquisa apresentou a existência de 36 patentes registradas em diferentes países, entre os anos de 2010 e 2015, apenas no setor farmacêutico. Entretanto, apesar do Brasil possuir uma das maiores biodiversidades do mundo (GANEN, 2010), solicitou registro de depósito de apenas cinco (05) patentes no Instituto Nacional de Propriedade Industrial (INPI).

Ressalta-se ainda a possibilidade de disponibilizar consultas online ao acervo pelo site http://www.isecensa.edu.br/laquibio, permitindo a trocar de informações entre profissionais e estudantes das áreas da micologia, botânica, ecologia e outras, no intuito de facilitar a identificação de fungos cultiváveis e, consequentemente, estimular e incentivar futuras pesquisas e parcerias a respeito de fungos endofíticos de restinga.

\section{CONCLUSÕES}

A manipulação de grande volume de dados gerados no armazenamento e manutenção de colônias de fungos requer o gerenciamento por meio da implantação de um banco de dados que viabilize sua utilização. A planilha eletrônica mostrou-se adequada para a manipulação dos dados da Micoteca do LAQUIBIO e a associação por fotodocumentação das colônias puras dos fungos são essenciais para o registro e a comparação, no tempo, dos isolados repicados. Acredita-se que esta iniciativa contribua para o conhecimento, a conservação e a divulgação do ecossistema restinga no qual estas espécies ocorrem.

\section{REFERÊNCIAS}

ABREU, M.M.V.; TUTUNJI, V.L. Implantação e manutenção da coleção de culturas de microorganismos do UniCEUB. Universitas Ciências da Saúde, v.2, n.2, p.236-251, 2004. DOI: 10.5102/UCS.V2I2.535.

ALFENAS, A.C., MAFIA, R.G. Métodos em fitopatologia. Viçosa: UFV, 2007. 382p.

ANDRADE, O.F., NUNES, M.S., TEIXEIRA, J.S.M., OLIVEIRA, M.M., BERBARA, R.L.L., SARAIVA, V.B. Fungos micorrízicosarbusculares (FMAs) em uma formação vegetal de restinga: ecologia e potencial para micorrizorremediação de hidrocarboneto do petróleo. VÉRTICES, v.17, n.3, p. 7-33, 2015.

AZEVEDO, J.L., MACCHERONI, W. JR., PEREIRA, J.O., ARAUJO, W.L. Endophytic microorganisms: a review on insect control and recent advances on tropical plants. Elect $J$ Biotechnol, 3:40-65, 2000.

BEZERRA, J.D.P., NOGUEIRA, E.B.S., CORREIA, A.C.R.B., SOUZA, S.C., PENNA, L.A.O., MACIEL, M.H.C., SILVA, L.R.C., GOMES, B.S., MAGALHÃES, O., NEVES, R.P., MOTTA, C.M.S. Micoteca URM da UFPE: uma fonte de recursos biológicos do Brasil. Anais da Academia Pernambucana de Ciência Agronômica, Recife, vols. 13/14, p.47-56, 2016/2017. 
CÂMARA, R. N., GRANATO, M., SA, M. R. As coleções microbiológicas e sua importância como patrimônio científico: o caso das coleções da Fiocruz. In: GRANATO, M., RANGEL, M.F. (org.). Rio de Janeiro: Cultura material e patrimônio da Ciência e Tecnologia. p.303-314, 2009.

DHANYA, N., PADMAVATHY, S. Impact of Endophytic Microorganisms on Plants, Environment and Humans. The Scientific World Journal, v. 2014, p. 1-11, 2014.

FREIRE, M.G.M., MUSSI-DIAS, V., COUTINHO, H.S., HERNANDEZ, G.A.F., TAVARES, L.P.S. Bioprospecção da flora fúngicaendofítica da reserva de Jurubatiba. Persp. Online: Biol. \& Saúde, v.6, n.21, p.6-13, 2016.

FREIRE, M.G.M., MUSSI-DIAS, V., SIQUEIRA, G.M.B., NASCIMENTO, D.F. O Tempo e a Restinga/Time and Restinga. 1. ed. Rio de Janeiro: RR Donnelley, v.1. 2015. 349p.

GANEN, S. R (org.) Conservação da biodiversidade: legislação e políticas públicas. Brasília: Câmara dos Deputados, Edições Câmara, 2010. 437 p. (Série memória e análise de leis; n. 2).

GOLDMAN, R.L., TALLIS, H. A critical analysis of ecosystem services as a tool in conservation projects: the possible perils, the promises, and the partnerships. Ann $\mathrm{N} Y$ AcadSci., 1162, p.63-78, 2009. DOI: 10.1111/j.1749-6632.2009.04151.x.

GONÇALVES, B., EDUARDO BASTOS, E., HANNA, S. Prospecção tecnológica de fungos endófitos e aplicações na indústria farmacêutica. Cad. Prospec., v.10, n.1, p.56-67, 2017. DOI: 10.9771/cp.v10i1.20114.

HYDE, K.D., SOYTONG, K. The fungal endophyte dilemma. Fungal Diversity, 33:163-173, 2008.

MUSSI-DIAS, V., COUTINHO, H.S., HERNANDEZ, G.A.F., TAVARES, L.P.S., FREIRE, M.G.M. Coleção biológica de fungos endofíticos das restingas do norte fluminense. VIII Congresso Brasileiro de Micologia. Florianópolis, Santa Catarina, Brasil. p.257, 2016.

OLIVEIRA-FILHO, A.T., FONTES, M.A.L. Patterns of floristic differentiation among Atlantic 531 Forests in southeastern Brazil and the influence of climate.Biotropica, v.32, n.4b, p.793$810,2000$.

PASSADOR, M.M., PIRES, G.C.C., FINATTI, D., APARECIDO, C.C., FIGUEIREDO, M.B. Divulgação técnica. Manutenção da viabilidade e patogenicidade de culturas mantidas na micoteca Mário Barreto Figueiredo. Biológico, v.72, n.1, p.51-55, 2010.

SANTOS, I.M., LIMA, N. Criteriafollowed in the establishment of a filamentousfungalculturecollection - Micoteca da Universidade do Minho (MUM). World JournalofMicrobiology\&Biotecnology, v.17, p.215-220, 2001.

SANTOS, T.T., VARAVALLO, M.A. Aplicação de microrganismos endofíticos na agricultura e na produção de substâncias de interesse econômico. Semina: Ciências Biológicas e da Saúde, v.32, n.2, p.199-212. 2011.

SILVESTRE, M.A. Bioprospecção de fungos com potencial para produção de $\beta$-glicosidase: avaliação dos parâmetros de cultivo e caracterização das enzimas produzidas. (Dissertação Mestrado em Ciência e Tecnologia Ambiental). Universidade Federal da Grande Dourados, MS. 2013. 26p.

VARGAS-ISLA, R., TIARA SOUSA CABRAL, T.S., ISHIKAWA, N.K. Instruções de coleta de macrofungosAgaricales e gasteroides. Editora INPA, 30 p, 2014. 
VIEIRA, G., PURIĆ, J., MORÃO, L.G., SANTOS, J.A., INFORSATO, F.J., SETTE, L.D., FERREIRA, H., SASS, D.C. Terrestrialand marine AntarcticfungiextractsactiveagainstXanthomonascitrisubsp. citri.LettAppl Microbiol. v.67, n.1, p.64-71, 2018. DOI: 10.1111/lam.12890. 